\title{
DEVELOPMENT OF RECRYSTALUZATION TEXTURE IN DEEP DRAWING STEELS, MEASURED BY X-RAY AND SINGLE ORIENTATION IN SEM
}

\author{
B. PLUTKA, H. P. HOUGARDY, Max-Planck-Institut für Eisenforschung \\ GmbH, Max-Planck-Str. 1, 4000 Düsseldorf, Germany
}

\section{INTRODUCTION}

In deep drawing steels the development of recrystallization was investigated by single orientation (SO) measurements by selected area electron-channeling pattern in a SEM 1) and X-ray measurements 2,3 ). By comparison of the results combined with metallographic investigations new aspects to the mechanism of recrystallisation were got.

\section{EXPERIMENTAL}

The materials investigated were melted as $100 \mathrm{~kg}$ units. The chemical composition is given in table 1 . The final hot rolling temperature was about $1100^{\circ} \mathrm{C}$. After rolling, the plates were cooled by water spray. Before cold rolling , the materials were heat treated, Tab. 1, to get a uniform grain shape. To guaranty a complete precipitation of $\mathrm{Ti}(\mathrm{CN})$ in alloy 2 annealing at $600^{\circ} \mathrm{C}$ 1h with furnace cooling was added. The texture of the hot band was only weak. The maximum density was not greater than $F(g) \quad 2.56$, the distribution was practically random. The texture after cold rolling by $70 \%$ was that usually got for deep drawing steels 4). The microstructure in both steels after cold-rolling was similar. After etching with nital, two different types of grains were visible, some showing a structure, in the following called 'rough grains' and other without a structure, called 'smooth grains'.

The results presented in the following are got by a recrystallization in a salt bath at $575^{\circ} \mathrm{C}$ for alloy 1 and $700^{\circ} \mathrm{C}$ for alloy 2 .

\section{RESULTS AND DISCUSSION}

Fig. 1a shows the appearance of the first recrystallized grains in cold deformed, rough grains. The new grains at first grow only into the rough grains, Fig. 1b. After 700 s of annealing all rough grains are recrystallized, the elongated smooth grains not. Fig. 1c shows the microstructure after complete recrystallization.

The texture after complete recrystallization for both steels can be compared with that of an interstitial free steel, Fig. 3a, c. In alloy 1 the Goss-orientation has a higher density than in alloy 2 . The $y$-fibre has a lower density in alloy 1 than in alloy 2.

Fig. $2 a$ shows the change of the density of some orientations with recrystallization time for steel 1. 'Start of recrystallization' (SR) is defined as the time, recrystallized grains are visible by light microscopy. Up to that time, there is no remarkable change in density. The densities of the $\alpha$-fibre orientations decrease rapidly, if the recrystallized grains start to grow into the cold worked smooth grains. After complete recrystallization the densities of $\{111\}<112>$ and $\{111\}<110>$ 
for steel 1 are slightly lower than in the cold-rolled state. For alloy 2 the densities for these orientations are similar after cold-rolling and after complete recrystallization. Fig. $2 \mathrm{~b}$ shows the change of micro hardness related to the hardness after complete recrystallization as a function of annealing time. After cold-rolling the microhardness in the rough grains is twice the value of the smooth grains. Before start of recrystallization the hardness in the rough grains already decreases remarkably.

For the determination of the orientation of single grains completely recrystallized specimens and specimens with recrystallized grains having a size just sufficient for measurement were selected.

For both alloys, in textures determined from single orientations, the density of the $y$-fibre shows greater differences between $\{111\}<112>$ and $\{111\}<110>$ than the texture measured by $X$-rays, Fig. 3a - d. All measurements of the single orientations were carried out on longitudinal sections. Only grains laying in the middle of the specimen in a band covering one third of the thickness of the specimen were measured. The pole figures determined by $X$-ray technique were measured parallel to the rolling plane in the middle of the specimen. In another steel was found 5$)$, that the relation of the densities of the $\{111\}<112\rangle$ and $\{111\}$ $<110\rangle$ components in the cold rolled state increases with the distance from the middle of the specimen. This may be the reason for the difference between textures from single orientation- and X-ray measurements. Textures determined by both methods show a comparable density along the $\alpha$-fibre for $\Phi=0$ - 40 . For both the alloys, grains belonging to the $y$-fibre have an average area which is about $20 \%$ greater than that of all the other grains. The largest grains belong to the orientation $\{111\}<112>$.

For partly recrystallized specimens neither for alloy 1 nor for alloy 2 the texture measured by single orientation and by $X$-rays can be compared, Fig. $4 a-d$. The texture measured by $X$-rays of this state is very similar to that determined after cold rolling. The measurement of single orientations determined only from recrystallized grains gives results which can be compared with that after complete recrystallization. For alloy 1 the density of the $y$-fibre after partial recrystallization is slightly higher than after complete recrystallization. The area fraction of grains belonging to the $y$-fibre in the partial recrystallized specimen is about $7 \%$ higher than after complete recrystalization. For alloy 2 the density of this fibre in the completely and partly recrystallized specimens are nearly the same.

The recrystallization of deep drawing steels has two steps. In step 1 rough grains recrystallize. The orientation distribution function (ODF) measured by $X$ rays is not changing, but the recrystallized grains have an ODF like that after complete recrystallization. In the second step the smooth grains recrystallize with a clearly decrease in the density of the $\alpha$-fibre measured by X-rays.

The results make clear, that if $25 \mathrm{vol} \%$ are recrystallized, the final texture is established. A decrease of density of the $\alpha$-fibre measured by X-rays for $\Phi=0$ $40^{\circ}$ can be observed, if the rough grains are recrystallized, and the new grains start to grow into the smooth grains. This is a hint, that the smooth grains have orientations belonging to the $\alpha$-fibre. On the other hand the rough grains must belong to the $y$-fibre, this is in agreement with results got by colour etching 6 ). Measurements of the hardness, Fig. $2 \mathrm{~b}$ show, that both types of grains recover, 
but this recovery does not initialize recrystallization in the smooth grains before other, already recrystallized grains grow into these areas. According to Figure $2 b$ the smooth grains have a hardness which is only $50 \%$ of that of the rough grains. TEM-investigations 7) of the size of dislocatic 1 cells are in agreement with this measurement. Grains with an $\{111\}$ orientation have smaller cells and by that a higher dislocation density than grains with $\{100\}$ or $\{112\}$ orientations.

In the partial recrystallized specimen new grains were found only in rough grains, which are at that time recrystallized up to aproximately $50 \%$. Assuming a random distribution of nuclei for recrystallization, there is no explanation for the fact, that at that time there is no change in the density of the $y$-fibre measured by $X$-rays. Also if there is a growth selection following a random nucleation, it is not probable, that this selection gives exactly the same orientation distribution function as before the start of the recrystallization. It must be assumed, that the first grains starting recrystallization have the same orientation as the rough grains. That means, that there are no large angle boundaries between the new grains and the cold rolled matrix. Recrystallization is defined as a large angle grain boundary motion. The observed process is a recovery or with other words recrystallization in situ. The new grains visible by light microscopy are just recovered grains, but further they should be called recrystallized grains.

Also the investigation of correlation between orientations of neighboured grains confirm that the observed process is a recrystallization in situ. The rotation axes as a function of the rotation angle are plotted in the standard triangle for a partial and complete recrystallized specimen of steel 1, Fig. 5a, b. A clustering of positions for the rotation axes is not visible. But there is a relative high number of rotation axes which have a rotation angle less than $20^{\circ}$. This becomes more clear, if the fraction of rotation angles is plotted as a function of the rotation angle, Fig. 6. For alloy 1, partly recrystallized, it is obvious, that for the real neighboured grains the fraction of rotation angles about $15^{\circ}$ is similar to that at $45^{\circ}$. For comparison, the rotation angle was calculated for 2000 orientation pairs. The orientation pairs were randomly formed from the orientations of grains of all specimens. For the real neighboured grains the fraction of rotation angles less than $25^{\circ}$ is remarkably higher than for random orientation pairs. As a consequence for high rotation angles the relations are opposite. For the partly recrystallized specimens the difference of the fraction of rotation angles with low angles between random and real neighboured grains are much greater than for complete recrystallized specimens. Coincidence grain bounderies were found very seldom. No correlation between the direction of rotation axes and the orientation of grains were found. Grains with difference in orientation less than $15^{\circ}$ form large areas with several grains. That means, that there exist extended volumes containing grains with similar orientations. Fig. 7 shows a microstructure of a partly recrystallized specimen. As far as the orientation of grains was determined, the boundaries are marked. The numbered grains have similar orientations, which are plotted in the (200) pole figures. For this representation, an area was selected with the maximum number of grains which could be found. The area contains $\mathbf{3 8}$ grains with an orientation about $(565)<1072>$. A detailed analysis shows, that the orientation differences between neighboured grains are not adding up. These results show again, that the recrystallized grains must keep the orientation of the cold rolled matrix because the probability is extremely low, that by random nucleation arrangements of many neighboured grains appear having similar orientations. 


\section{REFERENCES}

1) W. Lorenz, H. P. Hougardy, Texture, Vols. 8 and 9, (1988), pp. 579-598

2) J. Hirsch, M. Loeck, L. Loof, K. Kücke, Proceedings ICOTOM 7, Holland (1984), 765

3) H.J. Bunge, "Mathematische Methoden der Texturanalyse', Akademie Verlag, Berlin (1969)

4) U. v. Schlippenbach, Thesis, RWTH Aachen, (1987)

5) B. Plutka, Thesis, to be published, RWTH Aachen

6) R-Kern, Thesis, TU Clausthal, (1981)

7) R. L Every, M. Hatherly, Texture, Vol. 1, (1974), pp. 184-194

Tab. 1

Chemical composition, wt-\%

\begin{tabular}{|l|l|l|l|l|l|l|l|l|l|}
\hline Alloy & $\mathrm{C}$ & $\mathrm{Mn}$ & $\mathrm{P}$ & $\mathrm{S}$ & $\mathrm{N}$ & $\mathrm{Al}$ & $\mathrm{Si}$ & $\mathrm{O}$ & $\mathrm{Ti}$ \\
\hline 1 & 0.003 & 0.2 & $<0.003$ & $<0.003$ & $<0.001$ & 0.003 & $<0.003$ & & \\
\hline 2 & 0.004 & 0.2 & & & $<0.001$ & & & 0.004 & 0.1 \\
\hline
\end{tabular}

\begin{tabular}{|l|l|l|}
\hline Alloy & Heat treatment & Grain size $\mu \mathrm{m}$ \\
\hline 1 & $1000^{\circ} \mathrm{C} / 25^{\prime} /$ air $+1000^{\circ} \mathrm{C} / 20^{\prime} /$ /urnace & $160-180$ \\
\hline 2 & $1000^{\circ} \mathrm{C} / 20^{\prime} /$ hurnace $+600^{\circ} \mathrm{C} / 1$ h/furnace & $110-115$ \\
\hline
\end{tabular}

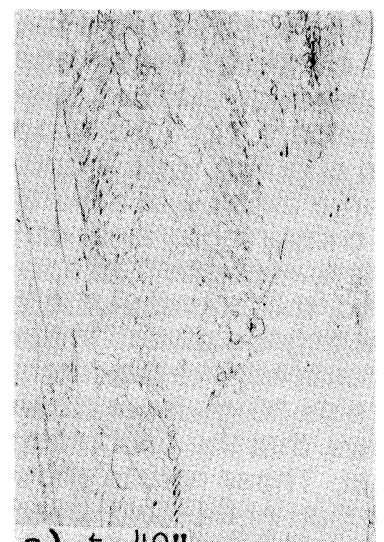

a) $t=40 "$
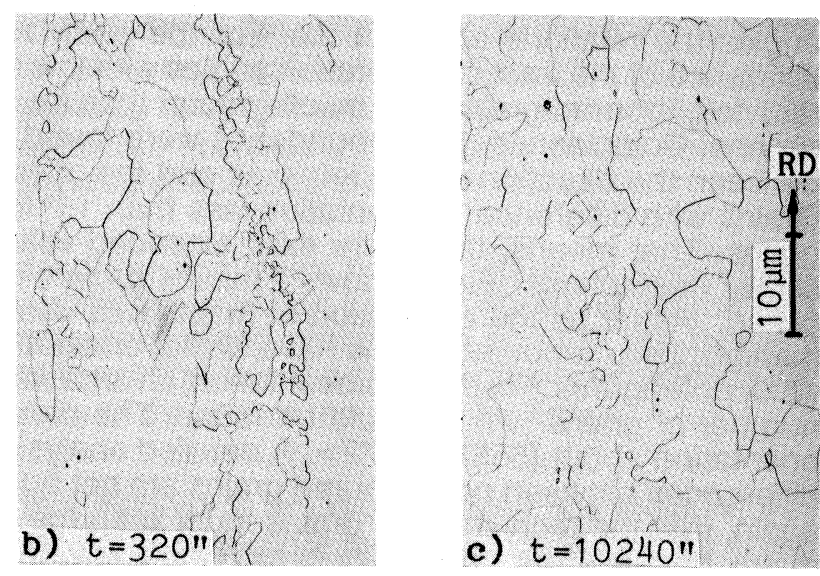

Fig. 1: Development of the microstructure during recrystallization at $575^{\circ} \mathrm{C}$, alloy 1 

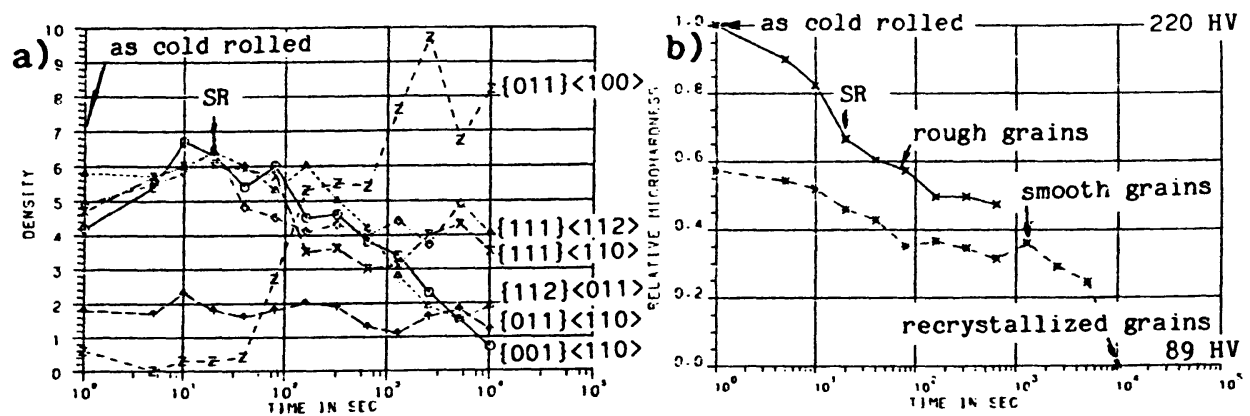

Fig. 2: Change of density for several orientations (a) and relative changes of micro-hardness (b) with recrystallization time at $575^{\circ} \mathrm{C}$, alloy 1
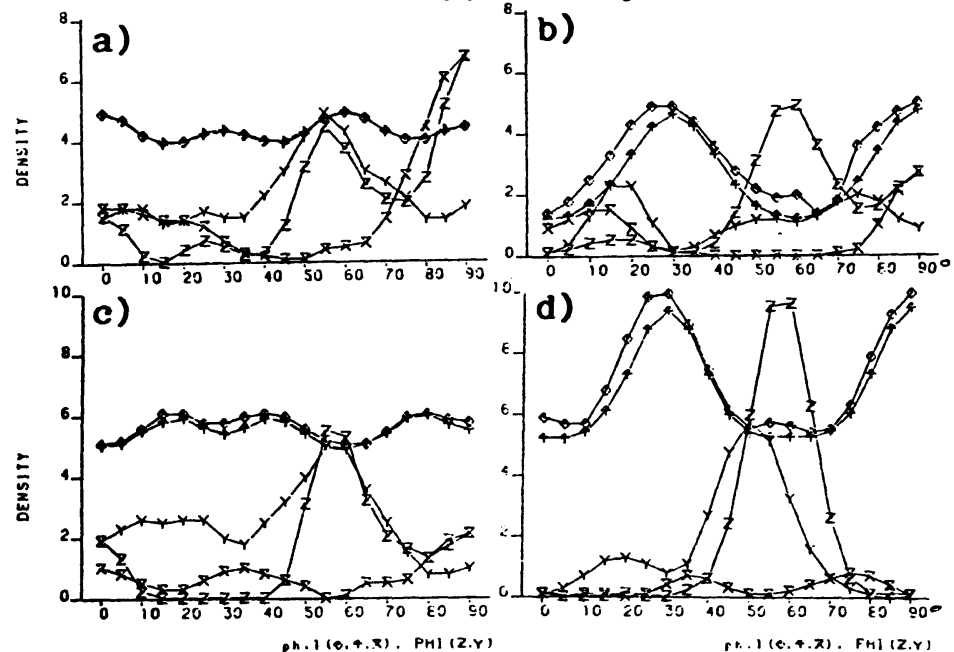

Fig. 3: Density along fibres, alloy $1(\mathrm{a}, \mathrm{b})$, alloy 2 , (c, d), both completely recrystallized.

Determined by $X$-rays $(a, c)$ and by $S O(b, d)$
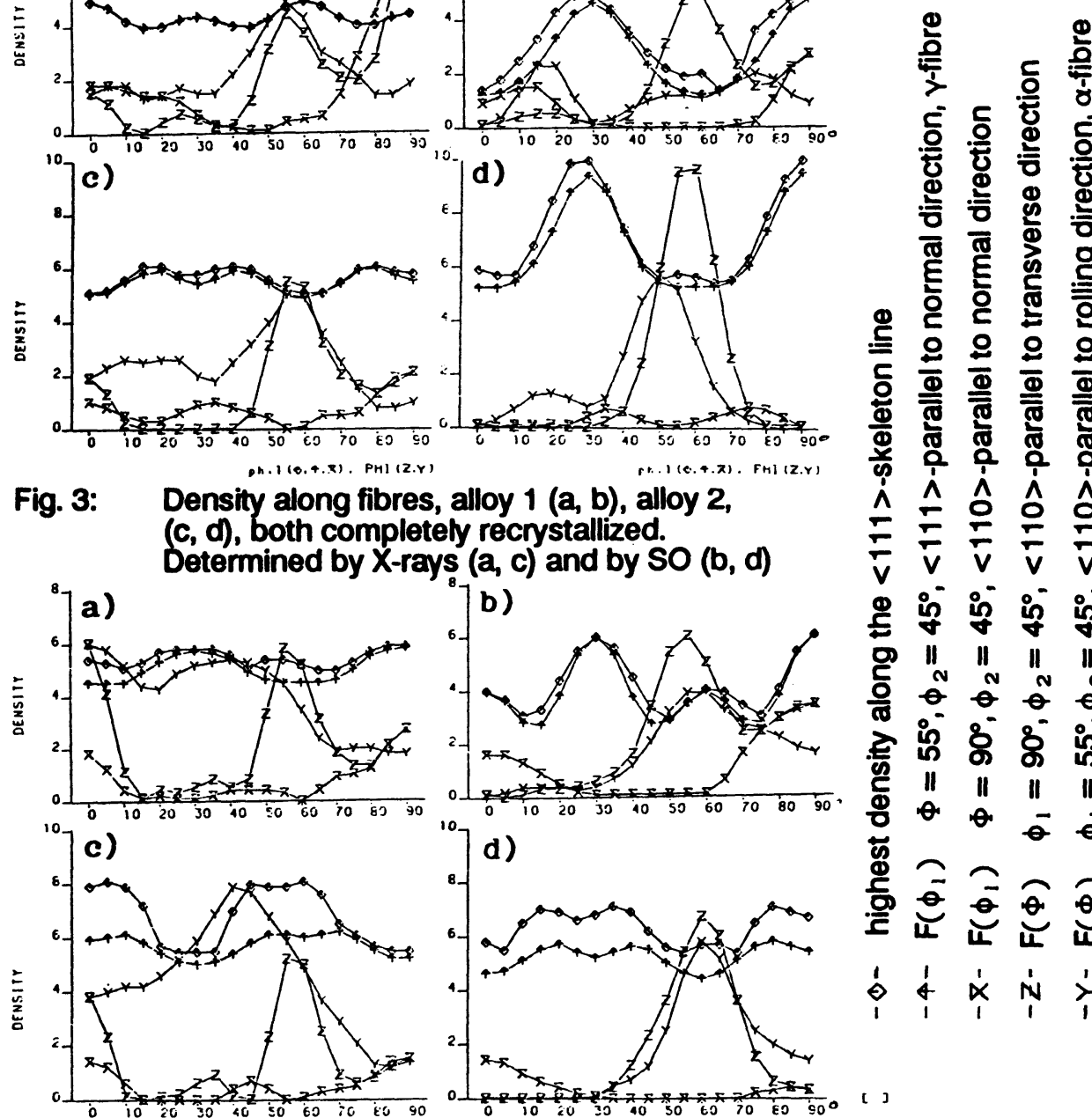

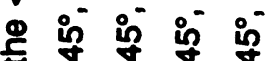

का "

을 $\hat{\theta}^{n} \hat{\theta}^{n} \hat{\theta}^{n}$

귱 \& \& \&

용

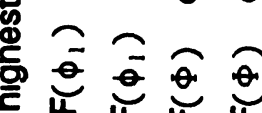

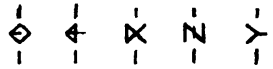

Fig. 4: Density along fibres, alloy $1(a, b)$, alloy 2, (c, d), both partly recrystallized. Determined by $X$-rays $(a, c)$ and by SO $(b, d)$ 
a)

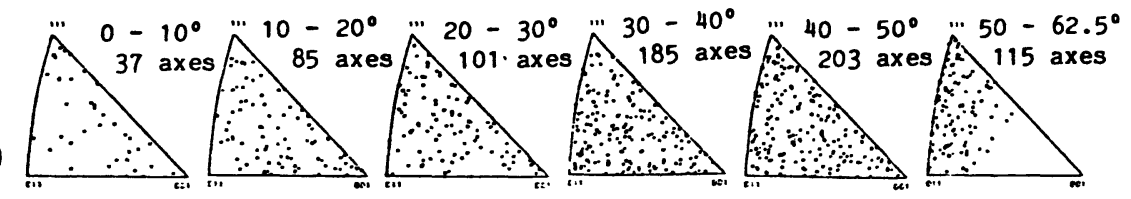

b)

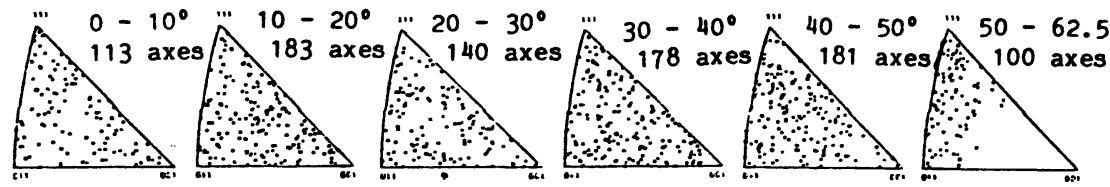

Fig. 5: Rotationaxes in the standard triangle as a function of the rotation angle, for complete (a) and partly (b) recrystallized alloy 1
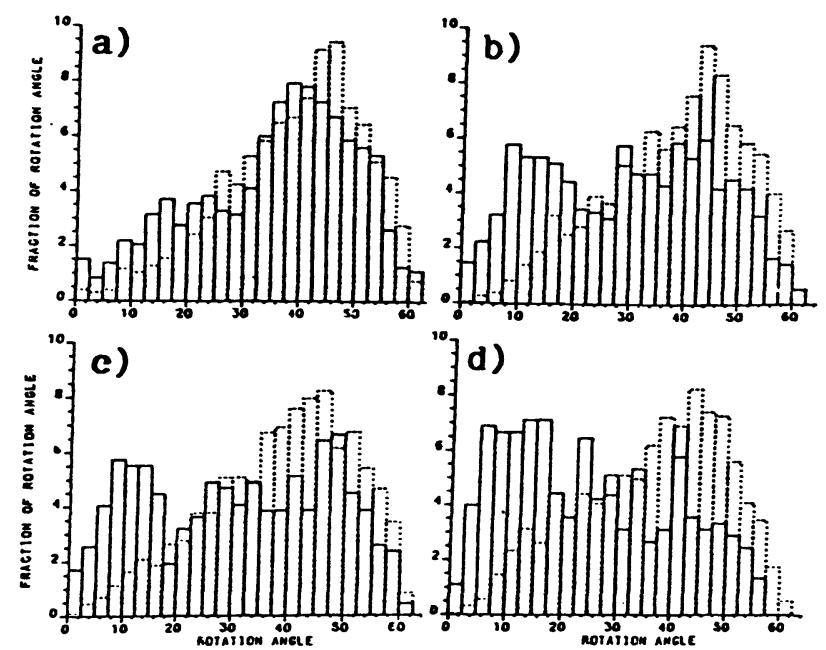

Fig. 6: Fraction of rotation angle as a function of angle. Alloy $1(a, b)$ and alloy $2(c, d)$, for complete ( $a, c)$ and partly $(b, d)$ recrystallized specimens. Solid line: as measured, dashed line: random orientation pairs
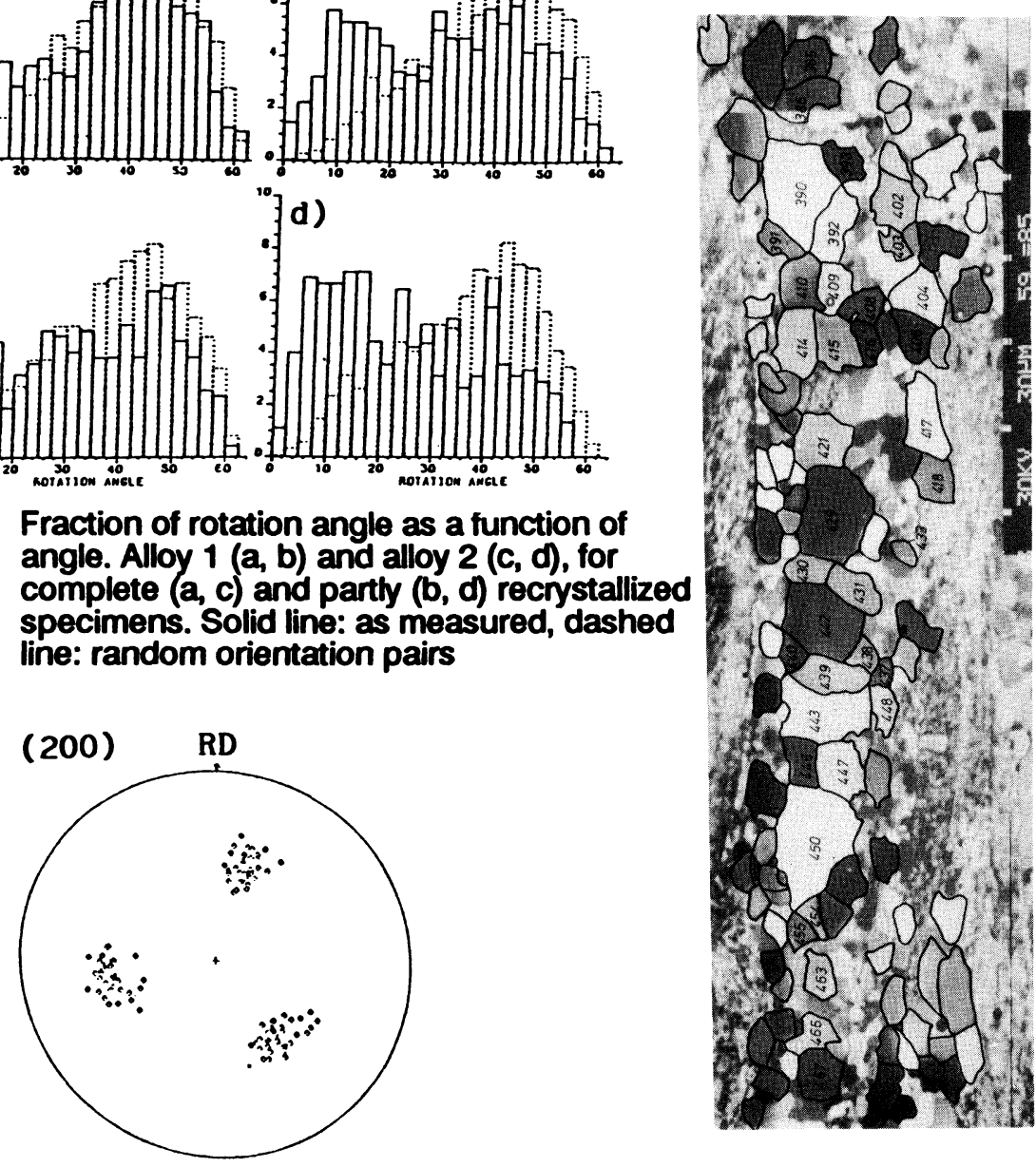

Fig. 7: Microstructure of a partly recrystallized specimen of alloy 2 measured by SO. Grains with number have similar orientations, plotted in the (200) pole figure. 